\title{
Feldeffektbasierte pH-Sensoren für die Überwachung von Kühlschmierstoffen
}

\author{
Frederic Güth, Pal Arki und Yvonne Joseph \\ Institut für Elektronik- und Sensormaterialien, TU Bergakademie Freiberg, Freiberg, Deutschland \\ Kontakt: queth@esm.tu-freiberg.de
}

\section{Zusammenfassung}

Feldeffektbasierte Elektrolyt-Isolator-Halbleiter (Electrolyte-Insulator-Semiconductor) EIS-Strukturen wurden auf ihre Eignung zur Überwachung der Degradation von Kühlschmierstoffen (KSS) hin untersucht. Eine Versuchsreihe unter Laborbedingungen wurde durchgeführt, um die Empfindlichkeit von Sensoren mit $\mathrm{Ta}_{2} \mathrm{O}_{5}$ als sensitiver Schicht auf durch Degradation verursachte $\mathrm{pH}$-Wert-Änderungen zu bestimmen. Des Weiteren wurden die Reproduzierbarkeit der Ergebnisse und mögliche Hystereseerscheinungen analysiert. Dabei wurde eine Sensitivität nahe dem theoretischen Maximum mit guter Wiederholbarkeit beobachtet. Die erzielten Resultate zeigen, dass die genutzten Sensoren in der Lage sind, verschieden stark degradierte KSS eindeutig voneinander zu unterscheiden. Daher eignen sich feldeffektbasierte EIS-Strukturen für den Einsatz in Sensorarrays, die zukünftig fähig sein sollen, Informationen über den Zustand von KSS während des Betriebes zu liefern. Dies würde eine Verbesserung der Kosteneffizienz und der Umweltbilanz des Einsatzes von KSS ermöglichen.

Schlagwörter: pH-Sensor, Kühlschmierstoffüberwachung, feldeffektbasierter chemischer Sensor

\section{Einleitung}

Kühlschmierstoffe (KSS) spielen eine entscheidende Rolle in spanenden Bearbeitungsverfahren. Neben den drei Hauptfunktionen Schmierung, Kühlung und Spanentfernung durch Spülung dienen KSS auch dem Korrosionsschutz der Anlage und des Werkzeuges [1, 2]. Es existiert eine Vielzahl von verschiedenen KSS, die jeweils an den gegebenen Anwendungsfall angepasst sind [3]. Um die genannten Aufgaben zu erfüllen und somit Anlagenverschleiß zu minimieren und die Prozessergebnisse $z u$ optimieren, muss die Zusammensetzung des KSS möglichst über den gesamten Einsatzzeitraum erhalten bleiben. Dem steht eine unvermeidliche Degradation während des Betriebes gegenüber. Ursachen hierfür sind vor allem der Eintrag von Fremdöl, gelösten Salzen und Metallspänen sowie der Austrag von wichtigen Inhaltsstoffen durch Verdunstung und Zersetzung. In wasserbasierten KSS sind insbesondere aus der Maschinenumgebung eingebrachte Mikroorganismen problematisch. Diese wandeln organische Additive in saure Abbauprodukte um, was neben dem Funktionsverlust des KSS auch zu erhöhten
Korrosionsproblemen führt. Der Degradation wird im laufenden Betrieb entgegengewirkt, indem verbrauchte Inhaltsstoffe nachgeführt werden, um Verluste zu kompensieren. Dieses Vorgehen erfordert eine möglichst exakte Überwachung des KSS online in der Anlage mit geeigneten Sensoren. Parameter wie Leitfähigkeit, Feststoffanteil und Ölkonzentration werden durch den Einsatz physikalischer Sensoren ermittelt und erlauben erste Rückschlüsse über den Degradationszustand des KSS. Entsprechend spezifische chemische Sensoren können weitere Informationen über Art und Konzentration von Ionen und organischen Substanzen liefern. Wünschenswert wäre die Integration verschiedener Sensoren in ein Array, das dann zur simultanen online Überwachung aller relevanten Parameter eingesetzt werden könnte.

Eine sehr wichtige Messgröße für die Einschätzung des Zustands von KSS ist der $\mathrm{pH}$ Wert. Zur Minimierung von Korrosion und Hemmung mikrobiologischer Prozesse wird dieser durch die Zugabe von Additiven auf 8,7 bis 9,3 eingestellt. Durch den Eintrag von Salzen und die beschriebene mikrobiologische Aktivität kann die Pufferkapazität eines KSS erschöpft werden, was 
sich durch einen abnehmenden pH-Wert äußert. Damit ist der $\mathrm{pH}$-Wert ein wichtiger Indikator für den Gesamtzustand von KSS [2]. Aktuell werden vor allem Glaselektroden und Indikatorpapier für die Überwachung des $\mathrm{pH}$-Werts in KSS eingesetzt. Damit verbunden sind Nachteile im Hinblick auf online Messungen in der Anlage. Indikatorpapier wird manuell durch Arbeiter an der Maschine ausgewertet und ist dadurch nicht für eine automatisierte und kontinuierliche Datennahme geeignet. Konventionelle Glaselektroden als potentiometrische Sensoren liefern zwar ein elektrisches Signal und sind somit in automatisierten Systemen prinzipiell einsetzbar, allerdings werden in der Praxis häufig Probleme durch eine beschleunigte Alterung der Elektroden aufgrund einer Verschmutzung durch die öligen Bestandteile des KSS beobachtet. Des Weiteren sind Glaselektroden konstruktionsbedingt nur schwer zu miniaturisieren und damit nicht in Sensorarrays zu integrieren. Alternative robuste und integrierbare $\mathrm{pH}$-Sensoren, die zugleich einen geringen Wartungsaufwand mit sich bringen, könnten entscheidend $\mathrm{zu}$ einer besseren Überwachung und damit einer effizienteren Nutzung von KSS beitragen, was neben den Verbrauchskosten auch die Umweltbelastungen durch die Entsorgung reduzieren würde.

Feldeffektbasierte $\mathrm{pH}$-Sensoren [4] erfüllen diese Anforderungen, wurden bislang aber noch nicht systematisch für den Einsatz in KSS untersucht. In dieser Arbeit werden die Ergebnisse erster Versuche mit Elektrolyt-Isolator-Halbleiter (EIS) Sensoren zur Überwachung von KSS präsentiert. Diese Sensoren zeichnen sich durch einen sehr einfachen Aufbau, der im einfachsten Fall aus einer dünnen Metalloxidschicht auf einem Halbleiterchip besteht, aus. Eine schematische Darstellung ist im eingefügten Nebenbild in Abb. 1 gezeigt. Das Wirkprinzip dieser Sensoren beruht auf der Wechselwirkung des KSS mit der $\mathrm{pH}$ sensitiven Metalloxidschicht des Sensors, die die Ausbildung eines vom $\mathrm{pH}-$ Wert abhängigen Oberflächenpotentials hervorruft (site-bindingModell [5]). Dieses Potential beeinflusst die Ladungsträgerverteilung in der Halbleiterschicht, was im Falle der EIS-Strukturen eine Verschiebung von gemessenen Kapazitäts-/Spannungskurven (C/V) verursacht. Da diese Verschiebung linear erfolgt und theoretisch der Nernst-Gleichung folgt, kann, wie in Abb. 1 gezeigt, eine Auslesekapazität definiert werden und die zugehörige Spannung als
Ausgangsgröße dieser $\mathrm{pH}$ Sensoren genutzt werden.

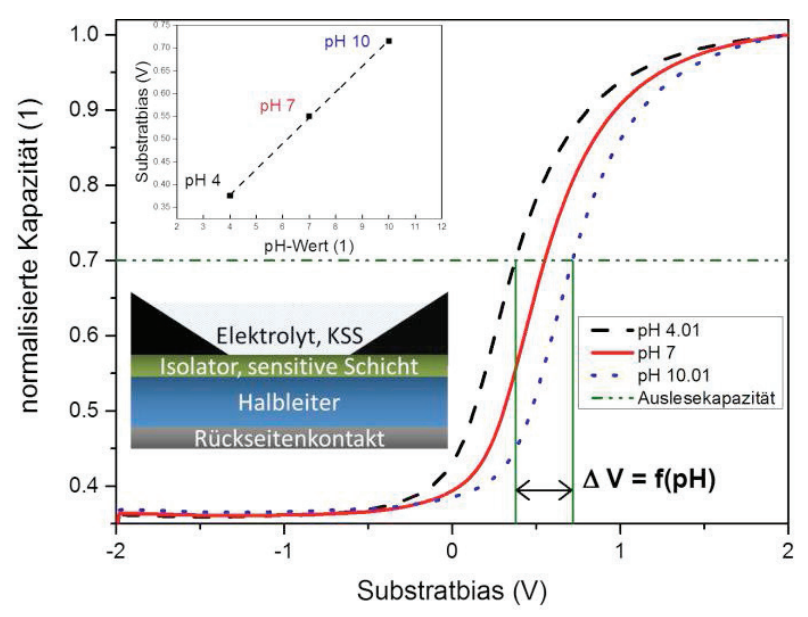

Abb. 1: Eine C/V-Kurvenschar aufgezeichnet bei verschiedenen $\mathrm{pH}-$ Werten mit einer EIS-Struktur. Die Verschiebung der Kurven in Abhängigkeit des $\mathrm{pH}-$ Wertes ist deutlich zu erkennen. In den eingefügten Nebenbildern ist schematisch eine EIS-Struktur sowie die lineare Abhängigkeit des Sensorsignals vom $\mathrm{pH}$-Wert abgebildet.

\section{Experimentelle Arbeiten}

\subsection{Materialien und Messaufbau}

Die für die Versuche genutzten EIS-Strukturen bestehen aus einer $160 \mathrm{~nm}$ dünnen $\mathrm{Ta}_{2} \mathrm{O}_{5}$-Schicht, die auf einen n-dotierten Siliziumwafer mit einem thermischen Oxid mit $55 \mathrm{~nm}$ Schichtdicke aufgebracht wurde. Den Rückseitenkontakt bildet eine Aluminiumschicht. $\mathrm{Ta}_{2} \mathrm{O}_{5}$ als $\mathrm{pH}$-sensitive Schicht wurde aufgrund der in der Literatur beschriebenen hohen Sensitivität und geringen Hysterese ausgewählt [6]. Drei 1,5 x 1,5 cm große Stücke, die aus dem gleichen Wafer gesägt wurden, wurden untersucht und werden im Folgenden als Sensor \#1, \#2 und \#3 bezeichnet. Als Analyt wurden wasserbasierte KSSEmulsionen genutzt, die freundlicherweise von ZF Friedrichshafen zur Verfügung gestellt wurden. Aus einem frischen Ansatz mit einem pH-Wert von 9,26 und einer nach langer Einsatzzeit verbrauchten Probe mit einem pH-Wert von 8,25 wurden durch Mischung künstliche Zwischenstufen der Degradation erzeugt. Die Messung von absoluten $\mathrm{pH}$-Werten erfolgte mit einer frisch kalibrierten kombinierten $\mathrm{pH}$-Elektrode.

Der verwendete Messaufbau ist in Abb. 2 dargestellt. In eine Messzelle mit den benötigten 
Zugängen für die Elektroden und die Fluidik wird die EIS-Struktur eingespannt. Der elektrische Messkreis wird durch eine $\mathrm{Ag} / \mathrm{AgCl}-$ Referenzelektrode, die den Vorderseitenkontakt durch die KSS herstellt und eine Kupferplatte, die gegen die leitfähige Rückseite der EIS-Struktur drückt, gebildet. Beide Elektroden sind durch geschirmte Kabel mit einem LCR-Meter (HP 4284A) verbunden, welches die C/V-Kurven aufnimmt. Die Bereitstellung, Mischung und Dosierung der auf $30{ }^{\circ} \mathrm{C}$ temperierten KSS-Proben sowie die Spülung des Systems erfolgt über Spritzenpumpen. Das gesamte System wird zentral durch ein LabVIEW-Programm gesteuert.

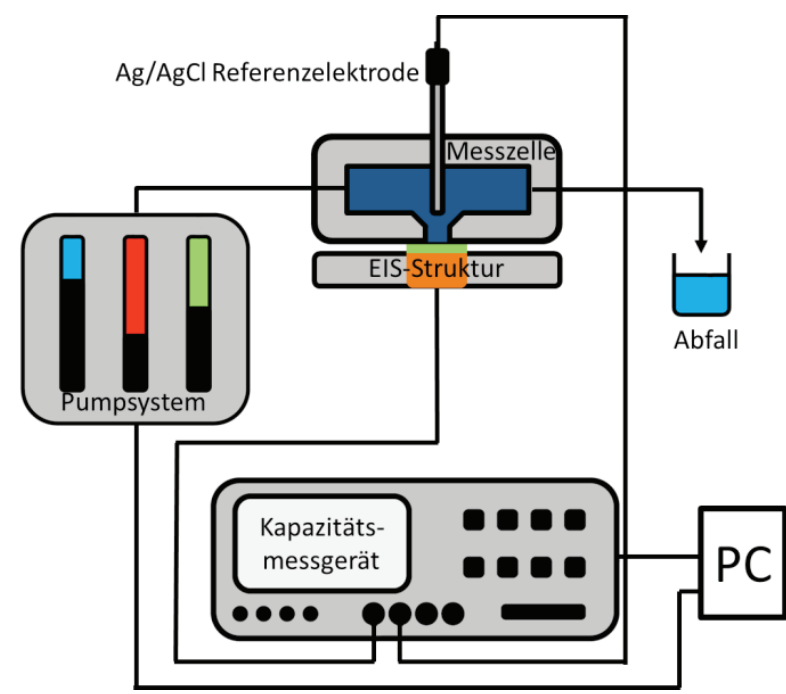

Abb. 2: Schematische Darstellung des Messaufbaus.

\subsection{Messablauf der Versuche}

Insgesamt wurden drei Versuche durchgeführt. Ein erstes Experiment (Versuch A) sollte die generelle Verwendbarkeit des Messprinzips demonstrieren und Auskunft über die Signaländerung bei

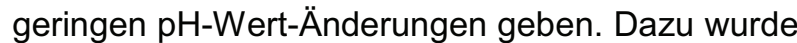
ausgehend von einem frischen Ansatz schrittweise verbrauchter KSS hinzugefügt, um die Degradation zu simulieren. Nach jedem Titrationsschritt wurden der $\mathrm{pH}-$ Wert des Elektrolyten sowie fünf $\mathrm{C} / \mathrm{V}$ Kurven aufgezeichnet, aus denen dann der Mittelwert und die Standardabweichung der Sensorantwort von Sensor \#1 bestimmt wurden.

In Versuch B wurden alle drei EIS-Strukturen der gleichen Messroutine unterzogen, um die Reproduzierbarkeit und Stabilität der Sensorantwort zu untersuchen. Für diesen Versuch wurde aus der frischen und der verbrauchten KSS eine 1-zu-1 Mischung als Zwischenstufe hergestellt. Beginnend mit der frischen Emulsion wurden die drei KSS-Proben in die Messzelle gepumpt und nach einer Wartezeit von zehn Minuten eine C/V-Kurve aufgezeichnet. Danach folgten im Abstand von je einer Minute vier weitere Messungen. Aus diesen insgesamt fünf Kurven wurden dann der Mittelwert und die Standardabweichung der Sensorantwort ermittelt.

Versuch C sollte Auskunft darüber geben, ob anhand der Sensorantwort die drei KSS-Zustände frisch, gemischt (1-zu-1) und verbraucht klar unterschieden werden können. Des Weiteren sollte eine mögliche Hysterese beim Wechsel von einer Probe zur nächsten ausgeschlossen werden. Dazu wurde die Messzelle zunächst mit dem frischen KSS gefüllt und nach fünf Minuten eine $\mathrm{C} / \mathrm{V}$-Kurve gemessen. Anschließend erfolgte ein Spülschritt mit deionisiertem Wasser gefolgt von der Befüllung mit der nächsten KSS-Probe. Nach weiteren fünf Minuten Wartezeit wurde dann wieder eine C/VKurve aufgenommen. Diese Prozedur wurde in der folgenden Abfolge wiederholt: frisch - gemischt frisch - verbraucht - frisch - gemischt - frisch verbraucht.

\section{Ergebnisse und Diskussion}

3.1 Versuch A: Nachweis des Konzepts und Messauflösung

Die Sensorantwort von Sensor \#1 in Abhängigkeit der zugegebenen Menge an verbrauchtem zu frischem KSS ist in Abb. 3 dargestellt. Wie erwartet nimmt der $\mathrm{pH}$-Wert mit zunehmender Degradation ab. Das Sensorsignal folgt diesem Verlauf in erster Näherung linear mit einer Steigung von 52,9 $\mathrm{mV} / \mathrm{pH}$ über den betrachteten Bereich von $0 \mathrm{Vol} \%$ verbrauchtem KSS (pH 9,26) bis hin zu $100 \mathrm{Vol} \%$ verbrauchtem KSS $(\mathrm{pH}$ 8,25). Die Standardabweichung an den jeweiligen Messpunkten beträgt weniger als $1 \mathrm{mV}$, was ca. $0,02 \mathrm{pH}-S c h r i t t e n$ entspricht. Damit sind alle betrachteten Volumenverhältnisse zwischen frischem und verbrauchtem KSS klar zu unterscheiden. Im Vergleich zu einer manuellen Überwachung der KSS mit Indikatorpapier kann daher davon ausgegangen werden, dass feldeffektbasierte EIS-Strukturen eine höhere Auflösung bieten und damit eine genauere Bestimmung des Degradationszustandes ermöglichen. 


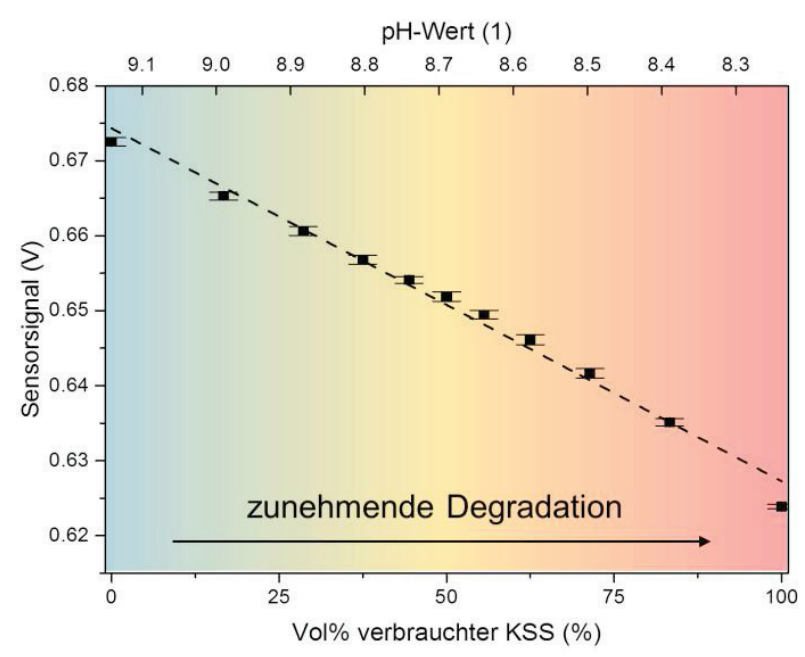

Abb. 3: Verlauf des Ausgangssignals von Sensor \#1 mit zunehmender Degradation des untersuchten KSS.

\subsection{Versuch B: Reproduzierbarkeit und Stabilität}

Die Ergebnisse von Versuch $B$ sind in Tab. 1 aufgelistet und in Abb. 4 dargestellt. Alle drei untersuchten EIS-Strukturen zeigen eine lineare Abhängigkeit des Sensorsignals von den abnehmenden $\mathrm{pH}$-Werten der verschieden stark degradierten KSS. Des Weiteren wurde an den jeweiligen Messpunkten für alle Sensoren eine geringe Standardabweichung des Signals von ca. $1 \mathrm{mV}(<0,02 \mathrm{pH})$ festgestellt. Dies zeugt von einem stabilen Messsignal und damit einem robusten Sensor. Allerdings umfasst der beobachtete Zeitraum nur knapp fünf Minuten und somit können keine Aussagen über das Langzeitdriftverhalten der Sensoren gemacht werden.

Durch die Messpunkte in Abb. 4 wurden Geraden gefittet, deren Steigung in Tab. 1 aufgelistet sind. Da mit den drei KSS-Proben nur drei Messpunkte und ein $\mathrm{pH}$-Bereich von einem $\mathrm{pH}$-Schritt betrachtet wurden, ist die Genauigkeit dieser Werte kritisch zu bewerten. Allerdings liegen alle Steigungen nah am durch die Nernst-Gleichung gegebenen theoretischen Maximum von 60,15 $\mathrm{mV} / \mathrm{pH}$ bei der Versuchstemperatur von $30^{\circ} \mathrm{C}$. Die
Abweichungen zwischen den Steigungen der drei Sensoren können neben der begrenzten Datengrundlage und damit verknüpften Fehlern auch über den manuellen Zusammenbau der Messzelle vor jedem Experiment begründet sein. Hierbei kann es zu Variationen des Elektrodenabstandes und des Probenvolumens über der Sensorfläche kommen. Dies ist auch eine wahrscheinliche Erklärung für den beobachteten Offset von Sensor \#2 (siehe Abb. 4) bei sonst gleichen Messbedingungen. Insgesamt zeigen alle drei EIS-Strukturen jedoch eine zufriedenstellende Übereinstimmung im Hinblick auf die Sensorantwort.

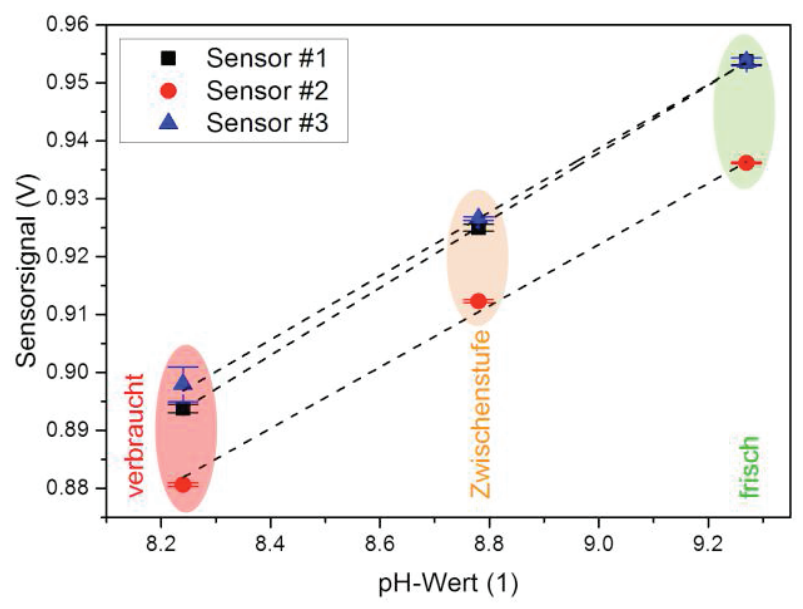

Abb. 4: Darstellung des Sensorsignals der drei untersuchten EIS-Strukturen bei Belegung mit KSS in drei verschiedenen Degradationszuständen.

3.3 Versuch C: Verhalten bei alternierender Eingangsgröße

Die Ergebnisse der Experimente mit wechselnder Belegung der Sensoren mit den drei KSS-Proben sind in Abb. 5 dargestellt. Zur besseren Vergleichbarkeit wurden die Messwerte auf das Sensorsignal der ersten Messung im frischen KSS normiert. Dies eliminiert die Verschiebung aufgrund der beschriebenen Unterschiede beim Zusammenbau der Messzelle.

Tab. 1: Ergebnisse von Versuch B. Neben den Sensorsignalen der drei EIS-Strukturen sind auch die pHWerte der KSS-Proben sowie die errechnete Empfindlichkeit der Sensoren über den betrachteten Bereich angegeben.

\begin{tabular}{|c|c|c|c|c|}
\hline & Frischer KSS & Zwischenstufe (1:1) & Verbrauchter KSS & \\
\hline $\mathrm{pH}$ & $9.26 \pm 0.01$ & $8.79 \pm 0.01$ & $8.25 \pm 0.01$ & Berechnete Empfindlichkeit \\
\hline Sensorsignal \#1 & $954 \pm 1 \mathrm{mV}$ & $925 \pm 1 \mathrm{mV}$ & $894 \pm 1 \mathrm{mV}$ & $58.2 \mathrm{mV} / \mathrm{pH}$ \\
\hline Sensorsignal \#2 & $936 \pm 1 \mathrm{mV}$ & $912 \pm 1 \mathrm{mV}$ & $881 \pm 1 \mathrm{mV}$ & $52.9 \mathrm{mV} / \mathrm{pH}$ \\
\hline Sensorsignal \#3 & $954 \pm 1 \mathrm{mV}$ & $927 \pm 1 \mathrm{mV}$ & $898 \pm 3 \mathrm{mV}$ & $55.1 \mathrm{mV} / \mathrm{pH}$ \\
\hline
\end{tabular}


Aus Abb. 5 kann entnommen werden, dass das Messsignal aller drei EIS-Strukturen den $\mathrm{pH}$ Werten der verschiedenen Degradationszustände folgt. Dabei kommt es zu keiner nennenswerten Hysterese, sodass die untersuchten Sensoren auch über längere Zeiträume mit zwischenzeitlicher Konditionierung der KSS durch Zugabe von verbrauchten Additiven und damit verbundenen $\mathrm{pH}$-Variationen verwendet werden können. Der Signalunterschied zwischen frischem und verbrauchtem KSS liegt bei mindestens $50 \mathrm{mV}$ und auch die Zwischenstufe ist mit einer Signaldifferenz von mindestens $20 \mathrm{mV}$ gut von beiden anderen Zuständen zu unterscheiden. Die Ergebnisse stimmen gut mit denen aus Versuch $B$ (siehe Tab. 1) überein. Das beschriebene Vorgehen könnte auch genutzt werden, um ein mögliches Sensorarray zur Überwachung von KSS direkt in der Anlage zu kalibrieren. Dazu würde vor einer Messung aus einem separaten Reservoir frischer KSS in der optimalen, nicht degradierten Zusammensetzung in den Sensor gepumpt und die jeweiligen Sensorsignale gemessen. Nach einer Spülung würde dann der zu untersuchende KSS eingeleitet und die Differenz der Sensorantworten bestimmt. Diese Methode würde zwar keine Absolutwerte der entsprechenden Parameter liefern, allerdings wären relative

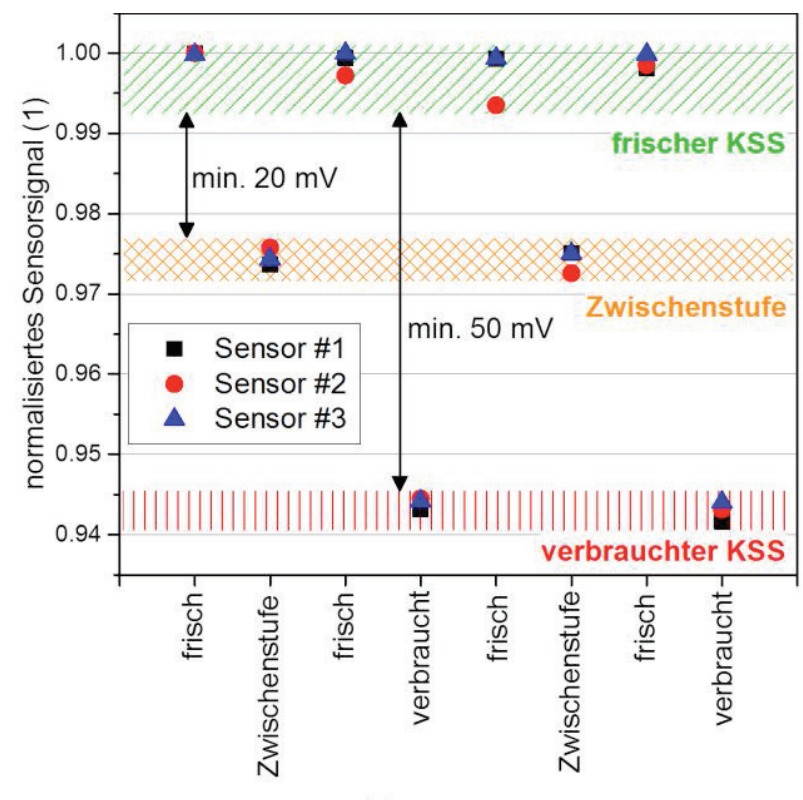

Messsequenz

Abb. 5: Ergebnisse von Versuch $C$ zur Ermittlung der Sensorantwort auf alternierende Belegung mit den drei KSS-Proben. Die Messabfolge ist auf der Abszisse angegeben.
Änderungen durch Degradation im KSS bestimmbar und Drifteinflüsse auf das Sensorverhalten könnten beschränkt oder eliminiert werden.

\section{Zusammenfassung und Ausblick}

Das Ziel dieser Arbeit war es, feldeffektbasierte $\mathrm{pH}$-Sensoren auf ihre Verwendbarkeit für die Überwachung des Degradationszustandes von wasserbasierten KSS hin zu untersuchen. Zu diesem Zweck wurden EIS-Strukturen hergestellt und einer Reihe von Experimenten unterzogen. Die untersuchten Sensoren zeigen eine Sensitivität nahe dem theoretischen Limit und eine hohe Reproduzierbarkeit der Messergebnisse mit geringen Fluktuationen. Daraus ergibt sich eine hohe Messauflösung mit zuverlässiger Unterscheidung von KSS in verschiedenen Degradationsstadien. Feldeffektbasierte $\mathrm{pH}$ Sensoren sind somit für den Einsatz in KSS geeignet und ihre Integration in entsprechende Überwachungssysteme könnte helfen, die Nutzungsdauer von KSS durch eine bessere Steuerung der Zugabe von Additiven zu verlängern. Damit wäre ein wichtiger Schritt hin zu einer effizienteren und damit kostensenkenden und umweltschonenden Nutzung von KSS gemacht.

Um die Vorteile von feldeffektbasierten $\mathrm{pH}$ Sensoren im Hinblick auf Miniaturisierung und Integration in Sensorarrays sowie mechanische Belastbarkeit nutzen zu können, muss auch die notwendige Referenzelektrode entsprechend robust sein und verkleinert werden. Dementsprechend ist es das Ziel zukünftiger Arbeiten, die bislang verwendete konventionelle $\mathrm{Ag} / \mathrm{AgCl}$-Referenzelektrode durch eine all-solidstate Alternative zu ersetzen. Des Weiteren sollen beide Elektroden auf einem Substrat vereint werden. Zudem muss das Langzeitdriftverhalten der Sensoren in KSS untersucht werden. Neben den EIS-Strukturen gibt es weitere Vertreter der feldeffektbasierten $\mathrm{pH}$-Sensoren, wie zum Beispiel den extended-gate Feldeffekttransistor (EGFET). Diese zeichnen sich besonders durch eine vergleichsweise einfache Integration der Ausleseelektronik aus, weshalb die hier durchgeführten Versuche mit EGFETs wiederholt werden. Parallel laufen auch Untersuchungen bezüglich verbesserter sensitiver Materialien für möglichst empfindliche und robuste Sensoren. 
Sobald das Gesamtpaket aus Sensorkonzept, sensitivem Material und Referenzelektrode hinreichend optimiert ist, werden Versuche an metallverarbeitenden Maschinen im laufenden Betrieb unter industriellen Bedingungen folgen.

\section{Literatur}

[1] KUCHARIKOVA, E.; PETERKA, J.: Cutting Fluids Management. In: Research Papers of the Faculty of Material Science and Technology, Slovak University of Technology , Bratislava. (2009), Nr. 26, S. $33-38$

[2] RAVE, A.; JOKSCH, S.: Monitoring metalworking fluids. In: ASTAKHOV, V.P.; JOKSCH, S. (Hrsg.): Metalworking fluids (MWFs) for cutting and grinding, first ed., Woodhead Publishing, Cambridge, 2012, S. 317 337

[3] EVANS, R.: Selection and testing of metalworking fluids. In: ASTAKHOV, V.P.; JOKSCH, S. (Hrsg.): Metalworking fluids (MWFs) for cutting and grinding, first ed., Woodhead Publishing, Cambridge, 2012, S. 23 - 78
[4] SCHÖNING, M.J.: "Playing around" with Field-Effect Sensors on the Basis of EIS Structures, LAPS and ISFETs. In: Sensors 5 (2005), Nr. 3, S. $126-138$

[5] YATES, D.E.; LEVINE, S.; HEALY, T.W.: Site-binding Model of the Electrical Double Layer at the Oxide/Water Interface. In: J. Chem. Soc. Faraday Transactions 1 (1974), Nr. 70, S. $1807-1818$

[6] MIKOLAJICK, T.; KÜHNHOLD, R.; RYSSEL, H.: The $\mathrm{pH}$-sensing properties of tantalum pentoxide films fabricated by metal organic low pressure chemical vapor deposition. In: Sensors and Actuators B: Chemical 44 (1997), Nr. 1-3, S. $262-267$

\section{Danksagung}

Die Autoren danken der ZF Friedrichshafen AG für die Bereitstellung der KSS-Proben. Die LabVIEWRoutine für die Kontrolle des Messaufbaus wurde von Markus Haverkamp (IESM, TU Freiberg) geschrieben. 